\begin{tabular}{cc} 
Sharif University of Technology \\
Scientia Iranica \\
SCIENTIA & Transactions B: Mechanical Engineering \\
I RAN I CA & \\
\hline
\end{tabular}

\title{
Identification of acceleration harmonics for a hydraulic shaking table by using Hopfield neural network
}

\author{
J. Yao*, T. Wang, Z. Wan, S. Chen, Q. Niu, and L. Zhang \\ College of Mechanical and Electrical Engineering, Harbin Engineering University, Harbin 150001, Heilongjiang, China.
}

Received 24 May 2016; received in revised form 31 August 2016; accepted 19 December 2016

KEYWORDS
Electro-hydraulic
shaking table;
Acceleration
harmonic;
Harmonic
identification;
Hopfield neural
network;
Real-time
performance.

\section{Introduction}

A shaking table, sometimes called shaking exciter or shaking generator, can be driven by pneumatics, electricity, hydraulics, piezoelectricity, etc. As a type of shaking tables, electro-hydraulic shaking tables are important test equipment for engineering research. It takes advantage of hydraulic control system and has been widely used in aerospace, automotive, construction, and many other important industrial fields, where generating strong force is needed [1]. Parallel mechanism is widely applied to hydraulic shaking tables. Take the world's largest shaking table, E-Defense in Japan, as an example. It has 5 and 14 hydraulic

\footnotetext{
*. Corresponding author.

E-mail address: travisyao@126.com (J.Yao)
}

actuators installed in parallel in each horizontal and vertical direction, respectively [2].

Hydraulic shaking tables are widely utilized to measure the response of scaled model structures subjected to base excitation to test their performances, such as reliability, fatigue, and lifetime. The specimen is fixed on the shaking table's platform, and desired signals are excited to the shaking table as the reference, usually in the form of acceleration. The shaking table is desired to reproduce the excitation signals with specified precision to simulate the mechanical condition encountered by the specimen. This is an effective way to measure the specimen's dynamic behavior by using feedback data.

A hydraulic shaking table is generally comprised of electrical and mechanical components, which have nonlinearities [3], such as dead zone in servo valve, backlash and friction between joints, friction in hydraulic cylinder, and saturation in electrical compo- 
nents. Sinusoidal vibration tests are usually employed to simulate periodic vibration. However, the shaking table's sinusoidal response is always inevitably distorted by harmonics, due to the system nonlinearities. THD (Total Harmonic Distortion) is usually applied as an index to determine the harmonic distortion. For a sinusoidal response with $n$-order harmonics, the THD can be expressed by:

$$
\mathrm{THD}=\frac{\sqrt{C_{2}^{2}+C_{3}^{2}+C_{4}^{2}+\cdots+C_{n}^{2}}}{C_{1}}
$$

where $C_{1}$ is amplitude of the fundamental response and $C_{i}$ is amplitude of the $i$ th harmonic, $i=2,3, \ldots, n$. It is discernible from Eq. (1) that the harmonic distortion can be considered as a relative measure of the harmonics' amplitudes as compared to the fundamental amplitude.

The harmonic distortion lowers the control performance in a hydraulic shaking table. It is an efficient way to reduce the THD by canceling harmonics, but requires the knowledge of the harmonic information. Thus, the prior knowledge about harmonics is essential to attain. The mostly used way to analyze harmonics is the FFT method, which transforms time-domain samples into the frequency-domain ones, but the phenomena such as aliasing, leakage, and picket fence effects, may result in inaccurate harmonic information. The estimated results are also averaged when using FFT. To overcome the shortages of employing FFT method for analyzing harmonics, researchers have developed new approaches and schemes to apply as an alternative method for harmonic identification to replace Fourier Transform based traditional techniques, especially in power systems.

Neural networks are utilized for harmonic analysis. Lin [4] used Back Propagation (BP) neural network algorithm to analyze dynamic harmonic distortion for a power system in noisy environments. Wang et al. [5] proposed a method by using BP network for accurate frequencies, amplitudes, and phases estimation for periodic signals of a power system, where the sampling frequency could not be locked on the actual fundamental frequency. Hamad et al. [6] introduced two multilayer feedforward neural networks to mitigate specific harmonic orders and, consequently, achieved low harmonic factor and reduced current THD for an active power filter. Abdeslam et al. [7] proposed a method based on Adaline networks for harmonic identification and compensation. Almaita and Asumadu [8] employed a radial basis function neural network to detect the fundamental, fifth, and seventh harmonic components in converter waveforms. Arabaci [9] studied a sensorless speed estimation method based on an artificial neural network for squirrel cage induction motors. Nascimento et al. [10] used artificial neural networks to estimate harmonics in load currents of single-phase systems.

Optimization algorithm is for finding an optimum for an objective function, which is defined over a given searching space; thus, many optimization algorithms have been applied for harmonic identification. Ulinuha et al. [11] proposed a hybrid genetic-fuzzy algorithm for optimal harmonic distortion control in distorted distribution systems with non-linear loads. Zamanan et al. [12] investigated a method using real coded genetic algorithm for identifying and tracking of harmonics in a power system. Xue et al. [13] developed a hyper spectral image classification approach by integrating the harmonic analysis, Particle Swarm Optimization (PSO), and support vector machine. Yin et al. [14] used PSO to estimate amplitude and phase of a harmonic. Yang and Wang [15] developed an analysis method based on improved Prony algorithm and PSO to improve the analysis precision for harmonic, interharmonic, and decaying DC component. Vasumathi and Moorthi [16] presented a hybrid adaptive neural network PSO algorithm for harmonic identification to improve its convergence rate and processing speed, and reduce the estimation error.

Adaptive signal processing technology has also been applied to identify harmonics. Ray and Subudhi [17] designed a nonlinear state estimation method by using ensemble Kalman filtering for estimating harmonics, inter-harmonics, and sub-harmonics. Singh et al. [18] used Kalman filter based on local ensemble transform to estimate the harmonic parameters of a power signal with harmonics, sub-harmonics, and interharmonics with white Gaussian noise. Jatoth and Reddy [19] studied a hybrid GA-adaptive PSO aided unscented Kalman filter for harmonic estimation of power system voltage/current waveforms. Ren and Kezunovic [20] studied a recursive wavelet transform based method to estimate frequency and harmonic parameters in a power system. Jain et al. [21] discussed a harmonics estimation method based on adaptive wavelet neural network for single-phase systems. Yang and Cheng [22] made use of wavelet transform to obtain a harmonic model, which was used to estimate the harmonic amplitudes and phase angles by solving the wavelet's coefficients and the scaling functions, and an on-line harmonic tracking method was also developed by combining Kalman filtering technique.

The above methods can get harmonic information, including amplitude and phase, but cannot directly obtain harmonic itself, which has to be synthesized by the identified harmonic's parameters, and they are generally focused on power systems. In this paper, we try to utilize Hopfield neural network to develop a harmonic identification scheme with good real-time performance to estimate acceleration harmonics in the 
shaking response for a hydraulic shaking table. This method can not only estimate harmonic's amplitude and phase, but also directly obtain the harmonic itself.

\section{The hydraulic shaking table}

The block diagram of the shaking table system is shown in Figure 1. It is a uniaxial shaking table with supply pressure of $8 \mathrm{MPa}$. A hydraulic cylinder is controlled by a two-stage servo valve with a natural frequency of $100 \mathrm{~Hz}$. The stroke of the cylinder is $50 \mathrm{~mm}$, and the diameters of its rod and piston are $\Phi 35 \mathrm{~mm}$ and $\Phi 40 \mathrm{~mm}$, respectively. The system is under acceleration control with a frequency bandwidth of $30 \mathrm{~Hz}$ to reproduce the desired shaking response.

The digital control system is based on an Advantech industrial computer, which includes I/O boards and is used as a real-time controller. The signal conditioner contains AD and DA conditioners for ana$\log$ transducer and servo valve control, respectively. The accelerometer is stuck on the shaking table's platform to obtain the acceleration response, while the displacement feedback is measured by a linear variable differential transformer, which is connected to the hydraulic cylinder.

The system controller is mainly composed of an input filter and a three-variable controller (TVC, shown in Figure 2) [23]. The input filter is used to transform the acceleration input into displacement command. TVC is commonly used as a basic controller to generate motion for hydraulic shaking tables [24]. The three variables of TVC refer to displacement, velocity, and acceleration of the electro-hydraulic shaking table. The feedback velocity is synthesized from the feedback displacement and acceleration. The TVC scheme can

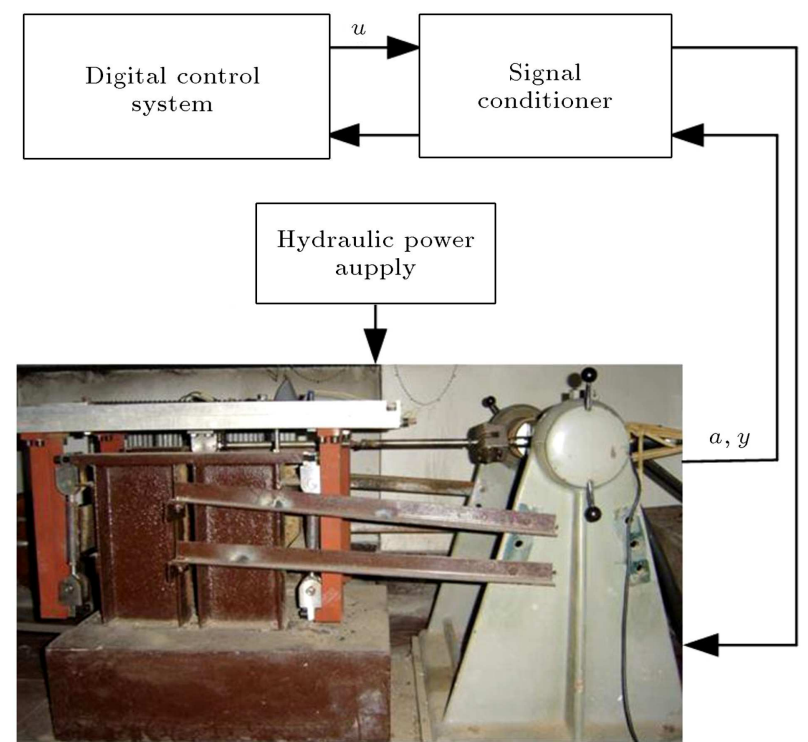

Figure 1. The hydraulic shaking table system.

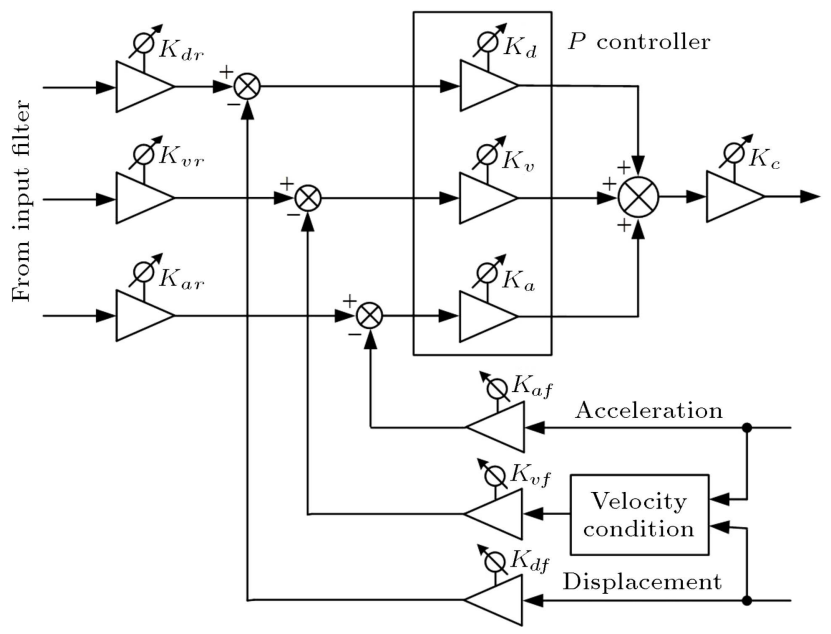

Figure 2. The block diagram of TVC.

Table 1. THD analysis results.

\begin{tabular}{cc}
\hline Item & Amplitude $\left(\mathbf{m} / \mathbf{s}^{2}\right)$ \\
\hline Fundamental response & 2.630 \\
2nd harmonic & 0.381 \\
3rd harmonic & 0.802 \\
4th harmonic & 0.320 \\
5th harmonic & 0.111 \\
6th harmonic & 0.019 \\
\hline
\end{tabular}

be divided into three parts: the feed-forward part, the feedback part, and the $P$ controller. $K_{d r}, K_{v r}$, and $K_{a r}$ are the feed-forward gains; and $K_{d f}, K_{v f}$, and $K_{a f}$ are the gains of the feedback part. The gains of the $P$ controller are $K_{d}, K_{v}$, and $K_{a} . K_{c}$ is the total gain. All of the gains are adjustable. TVC is an important controller for a hydraulic shaking table, since it can adjust the system robustness and disturbance suppression performance, and improve the reference tracking performance. The whole control program is achieved by using xPC Target, which is a real-time workshop in Matlab [25].

The system shaking response is shown in Figure 3 for the case when the acceleration input is $3 \sin (2 \pi \times 8 t)$ $\mathrm{m} / \mathrm{s}^{2}$. It can be seen that the shaking response is seriously distorted by higher harmonics, whose frequencies are integer multiples of the excitation frequency. The THD analysis (up to the sixth harmonic) is shown in Table 1. It can be clearly seen that the third harmonic is in dominance among higher harmonics. The value of THD is $36.21 \%$ calculated by Eq. (1).

\section{Harmonic identification scheme based on Hopfield neural network}

The Hopfield network, which is inspired by the brain neural network, is a well-known type of artificial neural network. It can be regarded as a model in 


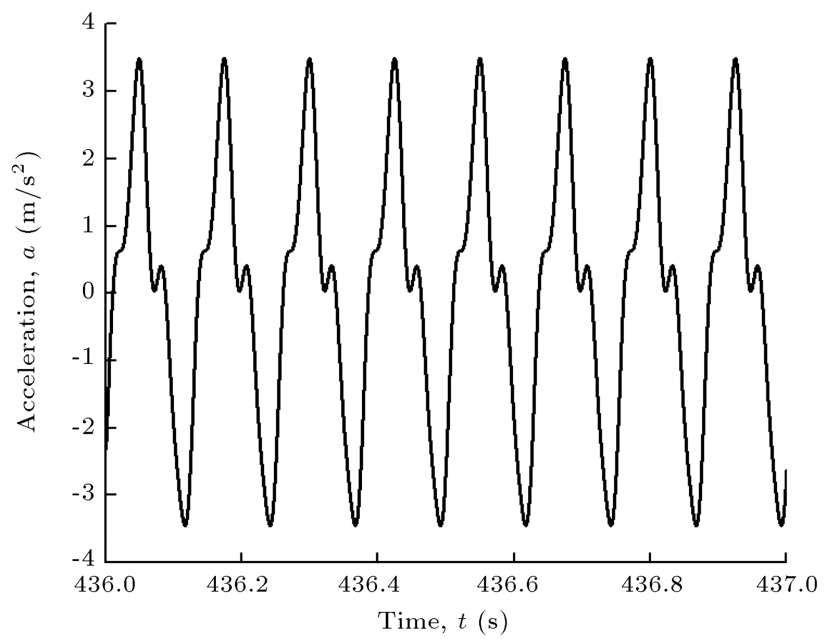

(a) Time-domain waveform

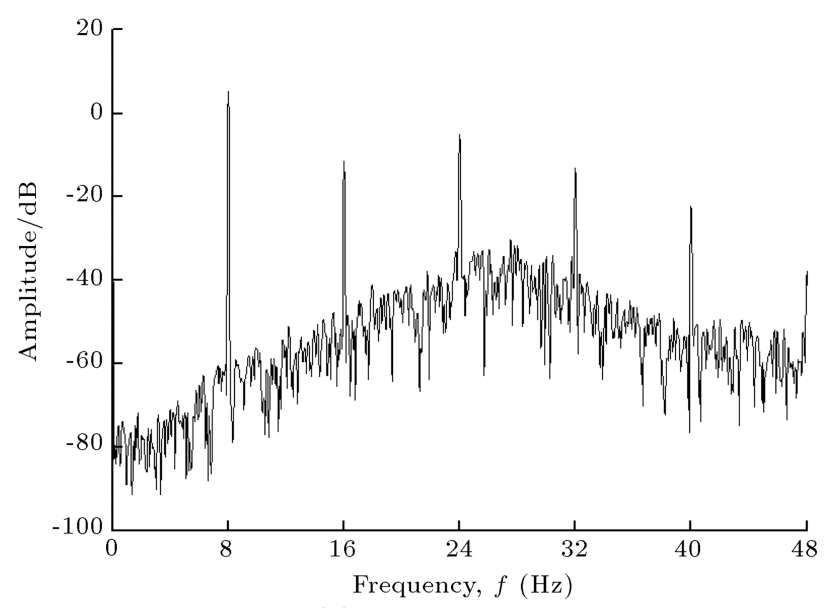

(b) Frequency spectrum

Figure 3. The system sinusoidal shaking response.

abstract neuron networks for memory and collective processing [26]. A Hopfield network needs to minimize an energy function. For an optimization problem, if its cost function can be mapped to the energy function, the network will approach a solution of the optimization. As a nonlinear dynamic system with fully connected recurrent artificial network, it has been extensively studied due to its wide applications in optimization, image reconstruction, signal processing, control, associative memories, information retrieval, and so on.

\subsection{Harmonic model}

The general model of a signal with unknown harmonics can be written as:

$$
x(t)=\sum_{l=1}^{n} X_{l} \sin \left(l \omega t+\phi_{l}\right)
$$

where $X_{l}$ and $\phi_{l}$ are the amplitude and phase of the $l$ th harmonic, respectively. $\omega$ is the fundamental frequency. Using notations $A_{k l}=X_{l} \cos \phi_{l}$ and $B_{k l}=X_{l} \sin \phi_{l}, X_{l}$ and $\phi_{l}$ can be computed by:

$$
\left\{\begin{array}{l}
X_{l}=\sqrt{A_{k l}^{2}+B_{k l}^{2}} \\
\phi_{l}=\arctan \frac{B_{k l}}{A_{k l}}
\end{array}\right.
$$

For a digital control system, let $T$ be the sampling time; Eq. (2) can be rewritten as:

$$
x\left(t_{k}\right)=\sum_{l=1}^{n}\left[A_{k l} \sin \left(l \omega t_{k}\right)+B_{k l} \cos \left(l \omega t_{k}\right)\right],
$$

where $t_{k}=k T, k=0,1,2, \ldots$

\subsection{Harmonic identification scheme}

The Hopfield network (illustrated in Figure 4) consists of a set of neurons and a corresponding set of unit-time delays, forming a multiple-loop system. The number of feedback loops is equal to the number of neurons. The output of each neuron is basically fed back via a unittime delay element to each of the other neurons in the network.

The Hopfield network iteratively determines the harmonics as well as the fundamental response by minimizing the error between the output of the network and the desired signal. The objective function is defined as:

$$
\xi_{k}=e^{2} / 2=\frac{\left[x\left(t_{k}\right)-d_{k}\right]^{2}}{2},
$$

where $d_{k}$ is the desired signal at time $t_{k}$. Substituting Eq. (4) into Eq. (5) yields:

$$
\xi_{k}=\left\{\sum_{l=1}^{n}\left[A_{k l} \sin \left(l \omega t_{k}\right)+B_{k l} \cos \left(l \omega t_{k}\right)\right]-d_{k}\right\}^{2} / 2 .
$$

To combine with the Hopfield network, let the objective function, $\xi_{k}$, be the energy function; thus, $E=\xi_{k}$. It is known that the energy function monotonously decreases and approaches a minimum value. If and only if $d E / d t=0, E$ is minimized, and an optimum solution can then be obtained.

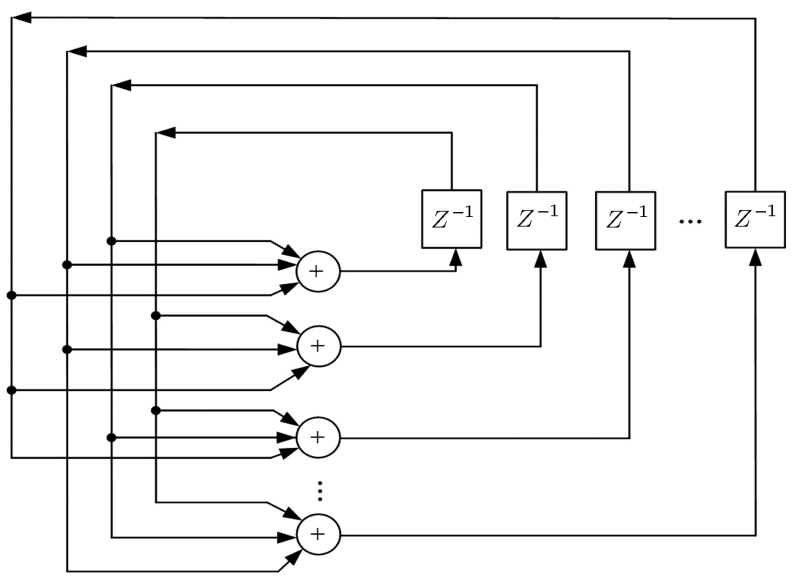

Figure 4. The Hopfield neural network. 


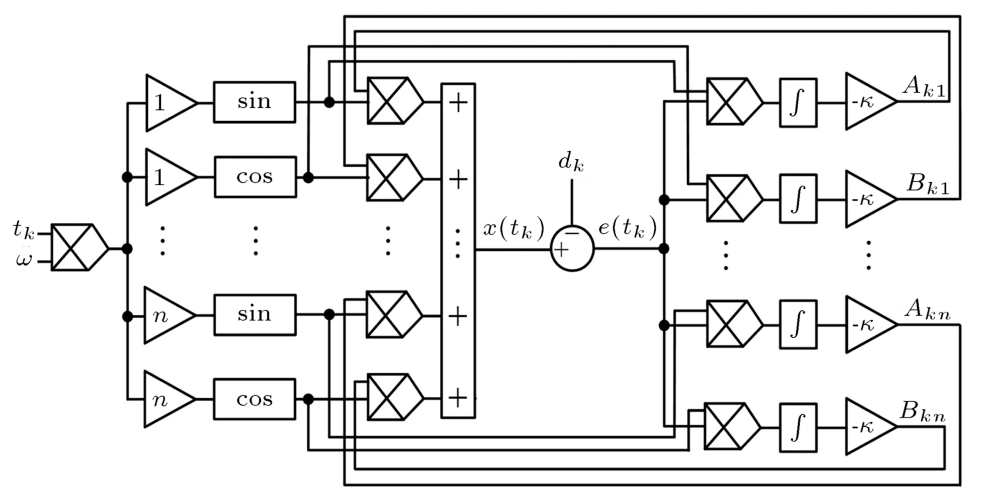

Figure 5. Block diagram of the proposed harmonic identification scheme.

The fundamental frequency, $\omega$, is usually known. For the $l$ th harmonic at time $t_{k}, A_{k l}$, and $B_{k l}$ are unknown and their coefficients are $\sin \left(l \omega t_{k}\right)$ and $\cos \left(l \omega t_{k}\right)$. Defining a vector as $\mathbf{X}_{k}=$ $\left[A_{k 1}, B_{k 1}, \ldots, A_{k n}, B_{k n}\right]^{T}$, and an input vector as $\mathbf{Y}_{k}=$ $\left[\sin \omega t_{k}, \cos \omega t_{k}, \ldots, \sin \left(n \omega t_{k}\right), \cos \left(n \omega t_{k}\right)\right]^{T}$, Eq. (6) can be written in a matrix form as:

$$
\begin{aligned}
\xi_{k}= & \left(\mathbf{X}_{k}^{T} \mathbf{Y}_{k}-d_{k}\right)^{2} / 2=\left(d_{k}^{2}-2 d_{k} \mathbf{Y}_{k}^{T} \mathbf{X}_{k}\right. \\
& \left.+\mathbf{X}_{k}^{T} \mathbf{Y}_{k} \mathbf{Y}_{k}^{T} \mathbf{X}_{k}\right) / 2
\end{aligned}
$$

According to the energy function theory (Martin, 2007), there is:

$$
\frac{d \mathbf{X}_{k}}{d t}=-\kappa \frac{\partial E}{\partial \mathbf{X}_{k}}=-\kappa \frac{\partial \xi_{k}}{\partial \mathbf{X}_{k}}
$$

where $\kappa$ is the gain. Taking partial derivative of $\xi_{k}$ with respect to $\mathbf{X}_{k}$ gives:

$$
\begin{aligned}
\frac{\partial \xi_{k}}{\partial \mathbf{X}_{k}}= & {\left[\frac{\partial \xi_{k}}{\partial A_{k 1}}, \frac{\partial \xi_{\mathrm{k}}}{\partial B_{k 1}}, \frac{\partial \xi_{k}}{\partial A_{k 2}}, \frac{\partial \xi_{k}}{\partial B_{k 2}}, \cdots,\right.} \\
& \left.\frac{\partial \xi_{k}}{\partial A_{k n}}, \frac{\partial \xi_{k}}{\partial B_{k n}}\right] .
\end{aligned}
$$

Differentiating $\xi_{k}$ with respect to $A_{k l}$ results in:

$$
\begin{aligned}
\frac{\partial \xi_{\mathrm{k}}}{\partial A_{k l}}= & \left\{\sum _ { j = 1 } ^ { n } \left[A_{k j} \sin \left(j \omega t_{k}\right)+B_{k j} \cos \left(j \omega t_{k}\right)\right.\right. \\
& \left.\left.-d_{k}\right] \sin \left(l \omega t_{k}\right)\right\} .
\end{aligned}
$$

Combining Eqs. (7)-(9) gives:

$$
\begin{aligned}
\frac{d A_{k l}}{d t}= & -\kappa\left\{\sum _ { j = 1 } ^ { n } \left[A_{k j} \sin \left(j \omega t_{k}\right)+B_{k_{j}} \cos \left(j \omega t_{k}\right)\right.\right. \\
& \left.\left.-d_{k}\right] \sin \left(l \omega t_{k}\right)\right\} .
\end{aligned}
$$

The unknown parameter, $A_{k l}$, can then be computed from:

$$
\begin{aligned}
A_{k l}= & -\kappa \int\left\{\sum _ { j = 1 } ^ { n } \left[A_{k j} \sin \left(j \omega t_{k}\right)+B_{k j} \cos \left(j \omega t_{k}\right)\right.\right. \\
& \left.\left.-d_{k}\right] \sin \left(l \omega t_{k}\right)\right\} d t .
\end{aligned}
$$

Applying similar procedure to $B_{k l}$, its value can then be solved by using:

$$
\begin{aligned}
B_{k l}= & -\kappa \int\left\{\sum _ { j = 1 } ^ { n } \left[A_{k j} \sin \left(j \omega t_{k}\right)+B_{k j} \cos \left(j \omega t_{k}\right)\right.\right. \\
& \left.\left.-d_{k}\right] \cos \left(l \omega t_{k}\right)\right\} \mathrm{d} t .
\end{aligned}
$$

The above harmonic estimation can be represented by Figure 5. The Hopfield network is an adaptive feedback network. The weights of the Hopfield network are $A_{k 1}, B_{k 1}, \ldots, A_{k n}$, and $B_{k n}$, which also denote the unknown harmonic parameters, including amplitudes and phases. The signal $x\left(t_{k}\right)$ can be treated as the estimated signal. The network's outputs, $A_{k 1}, B_{k 1}$, $\ldots, A_{k n}$, and $B_{k n}$, are fed back to the network and used as the weights to minimize the error such that the network can converge on its steady state. The algorithm does not need offline gradient estimations or data repetitions. The desired weight vector approaches an optimal solution when adapting to the adaptive algorithm.

\section{Simulation results}

Simulations are carried out to verify the proposed harmonic identification scheme. The input signal is $y=2 \sin (\omega t+1)+0.5 \sin (2 \omega t+0.8)+0.4 \sin (3 \omega t+0.5)+$ $0.2 \sin (4 \omega t+0.2)+0.1 \sin (5 \omega t+0.1)+0.06 \sin (6 \omega t+0.3)$. It contains 6 harmonics from the fundamental with frequency of $5 \mathrm{~Hz}$ to the sixth harmonic; thus $n=6$. During simulations, its sampling time is $1 \mathrm{~ms}$ and all initial values are set to zero, and $\kappa=4$. The 


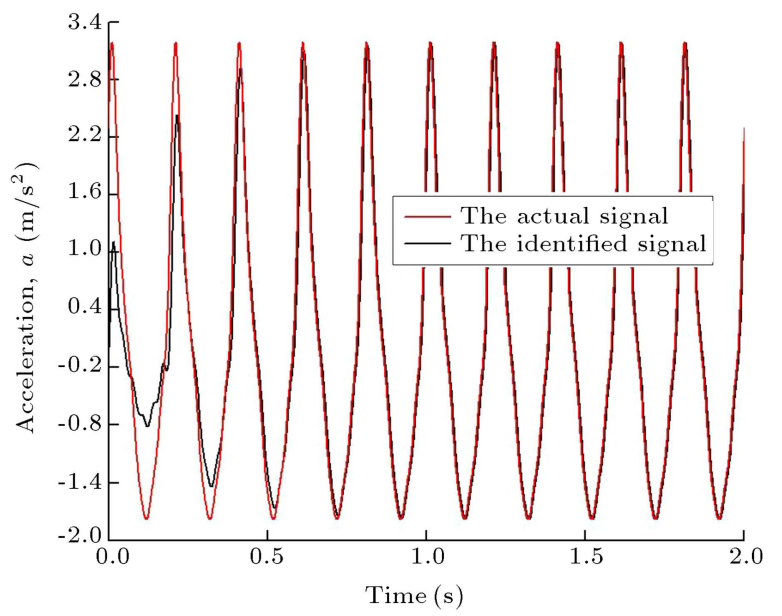

Figure 6. The identified acceleration plotted with the actual signal.

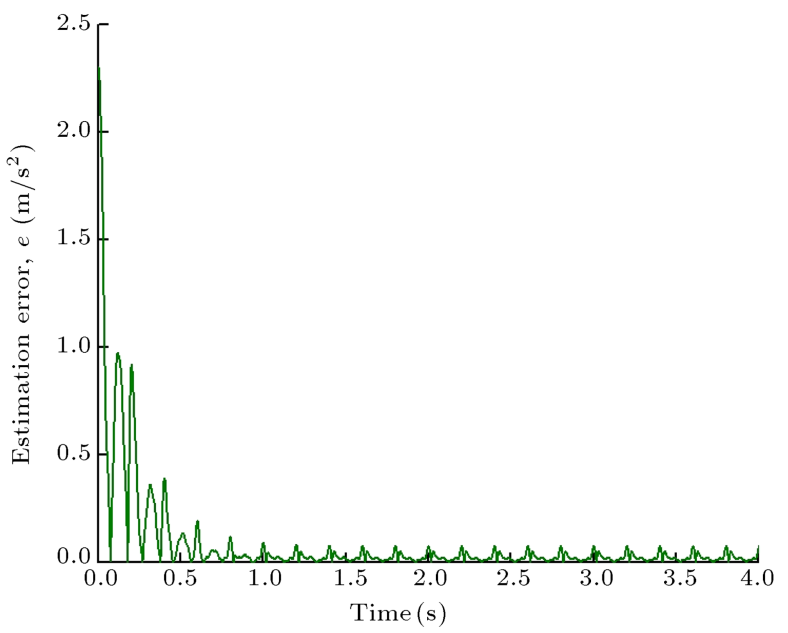

Figure 7. The estimation error between identified signal and actual signal.

identified acceleration is plotted with the input signal in Figure 6, from which it can be clearly seen that the identified signal asymptotically approaches the input signal within $1.5 \mathrm{~s}$.

Figure 7 shows the estimation error, which demonstrates that there is identification error at the beginning of the estimation, but the error is rapidly decreased and remains within a very small range after $1.5 \mathrm{~s}$. Figure 8 shows each harmonic, which is directly obtained from the method, while each harmonic's amplitude and phase are shown in Figure 9. The steady state values of the identified amplitudes and phases are nearly same as their nominal values. The simulation results indicate that the developed harmonic identification has good estimation accuracy and convergence.

\section{Experimental results}

The developed identification scheme is embedded in the real-time controller shown in Figure 1. The harmonic
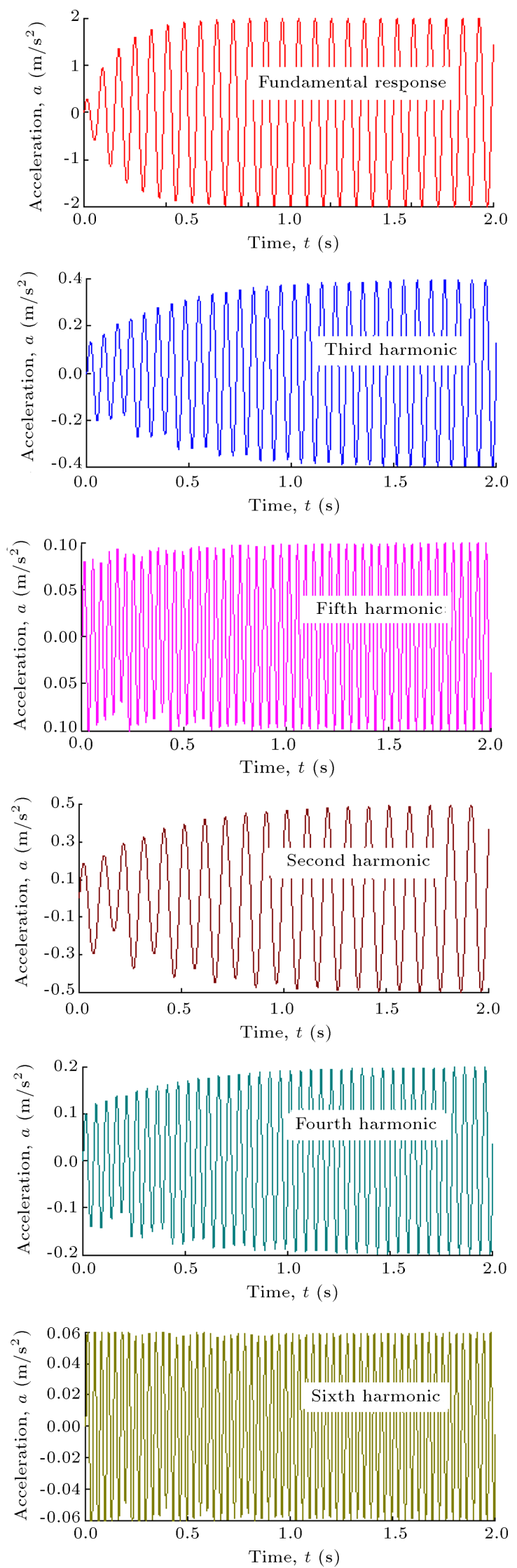

Figure 8. Each identified harmonic including the fundamental response. 

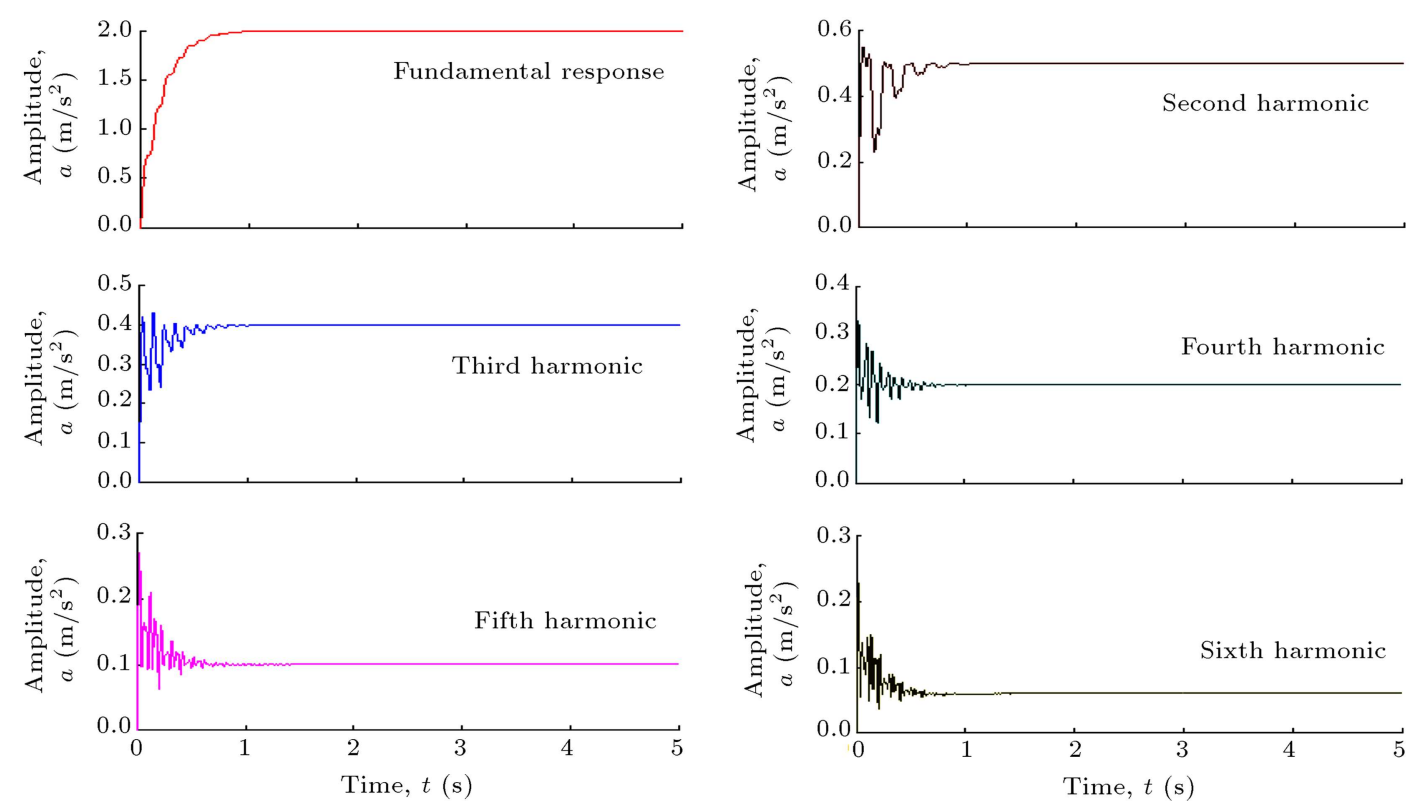

(a) Amplitude of each harmonic
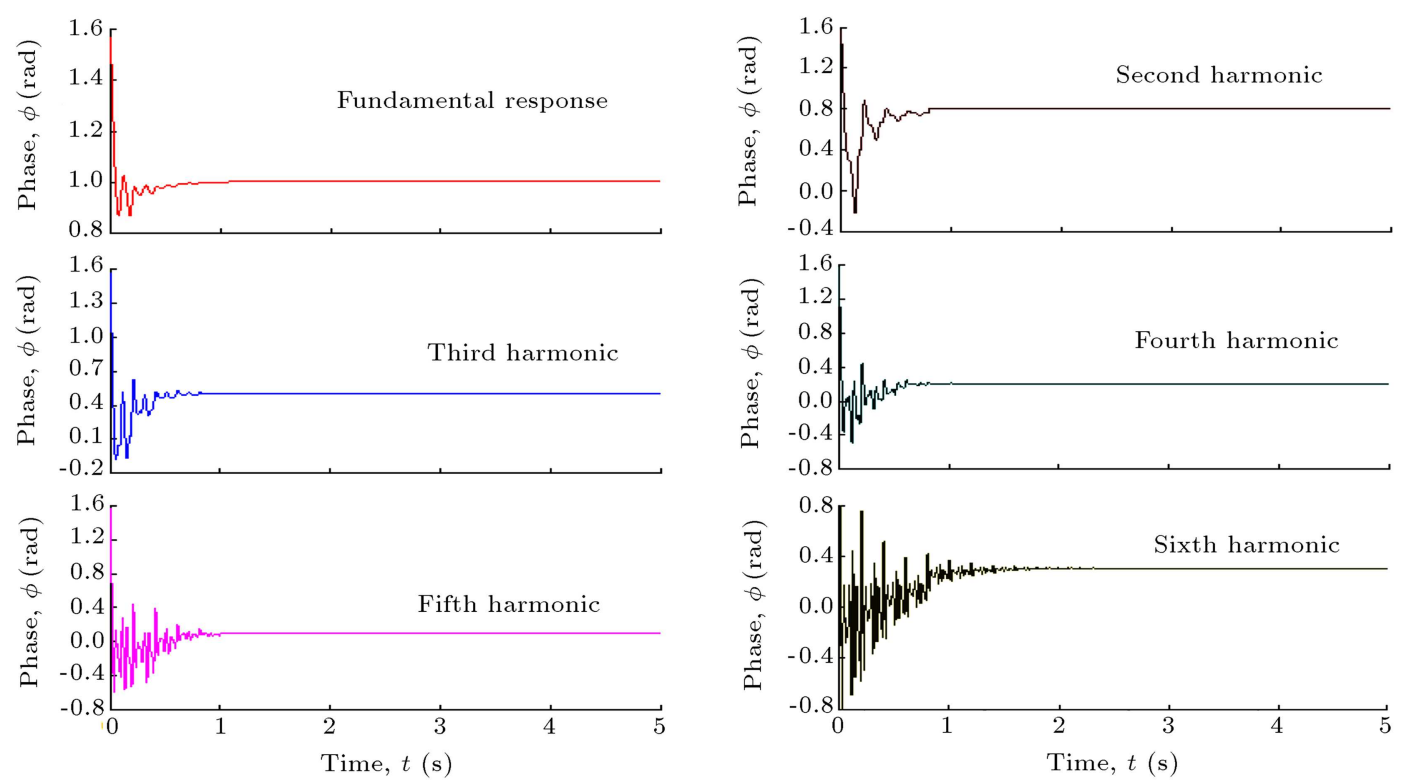

(b) Phase of each harmonic

Figure 9. Amplitude and phase of each harmonic.

identification algorithm, as well as the original controller, runs in the control system. When sinusoidal shaking tests are performed on the shaking table in Figure 1, the shaking response can be measured. The developed identification scheme is applied to the hydraulic shaking table to identify the harmonics in the sinusoidal shaking response. When the input acceleration is $3 \sin (2 \pi \times 8 t) \mathrm{m} / \mathrm{s}^{2}$, based on Figure 3 , six harmonics, including the fundamental, occur in the shaking response, so $n=6$. All of the initial values of the scheme are set to zero, and $\kappa=5$. The system sampling time is $1 \mathrm{~ms}$, and the desired signal is the actual shaking response shown in Figure 3(a).
The estimated acceleration and the actual acceleration are plotted together in Figure 10. It can be seen that the estimated signal asymptotically approaches the actual signal within $1.5 \mathrm{~s}$ with high precision. This means that high estimation precision can be obtained both in amplitude and in phase.

The identification error is shown in Figure 11. At the beginning of the identification, there is large estimation error. As the Hopfield network begins to train its weights, the error is decreased rapidly. When the weights converge, the error becomes very small and, finally, stays around the ideal error of $e=0$.

Each harmonic can be directly estimated; the 


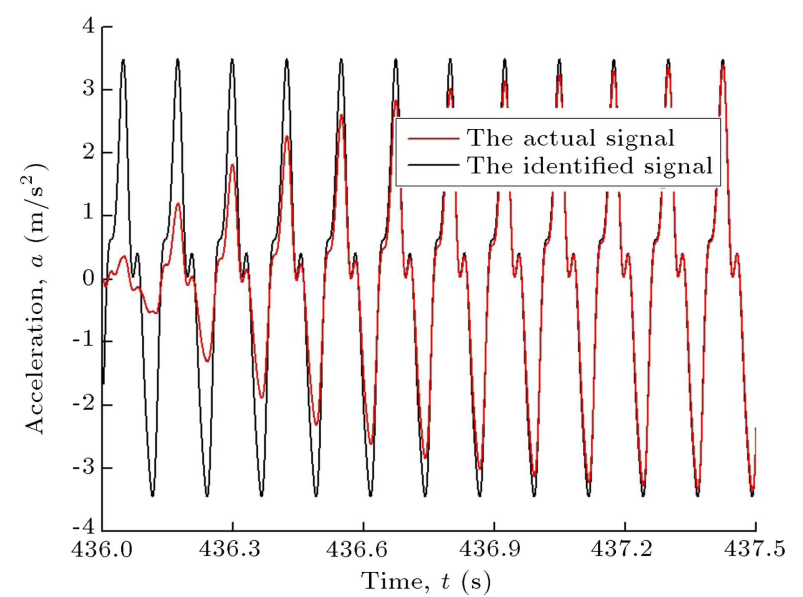

Figure 10. The estimated signal and the actual signal.

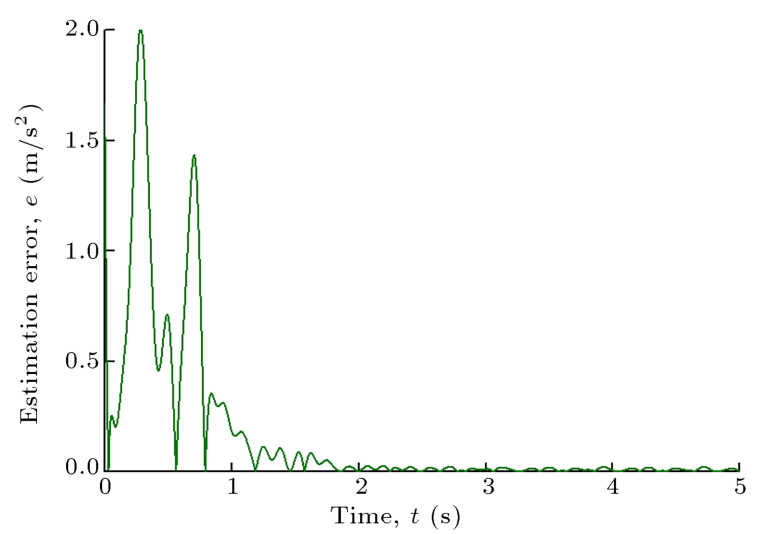

Figure 11. The experimental identification error between the identified signal and the actual signal.
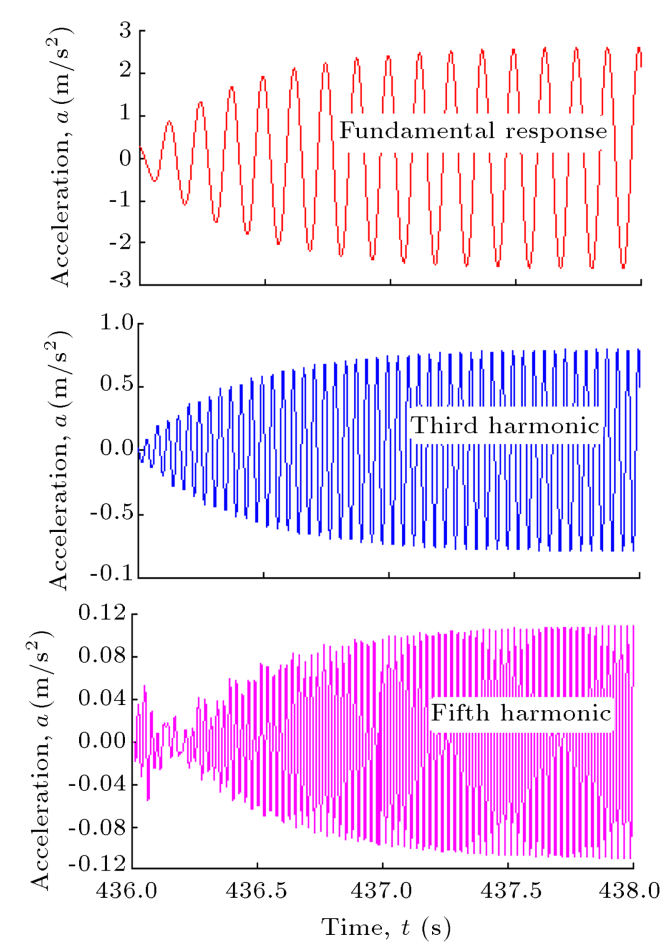

results are shown in Figure 12. It can be seen that harmonics are all well estimated and they converge. The amplitude and phase of each harmonic can be computed by Eq. (3); the results are shown in Figure 13. Compared with the amplitude values in Table I, which are calculated off-line by FFT, the estimated amplitudes well match the FFT-computed amplitudes. Though the sixth harmonic is smaller than any other harmonic, that is it is dominated by other harmonics, good estimation is also achieved for the smallest harmonic.

\section{Comparison results}

It is more convincing to give comparison with another method. The Recursive-Least-Squares (RLS) is an adaptive filter, which recursively finds the coefficients by minimizing a weighted linear least squares cost function. RLS-based estimation approaches have been successfully developed for accurate estimation of harmonics in power system signals [27-30]. This section compares the proposed method with RLS-based method. Figures 14 to 16 show the comparison results.

In Figures 14 to 16, it can be seen that RLSbased estimation method has large estimation error and is very oscillatory at the beginning of the estimation, though it exhibits faster convergence than the developed harmonic identification does; however, the latter has better estimation accuracy, and it can asymptotically track the harmonic parameters with smaller oscillation.

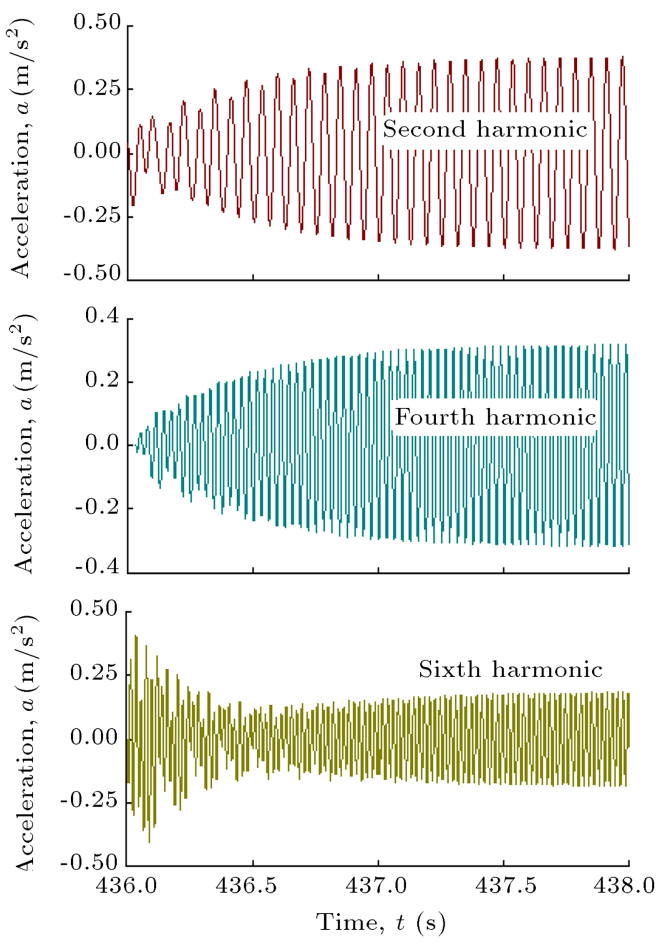

Figure 12. The identified harmonics. 

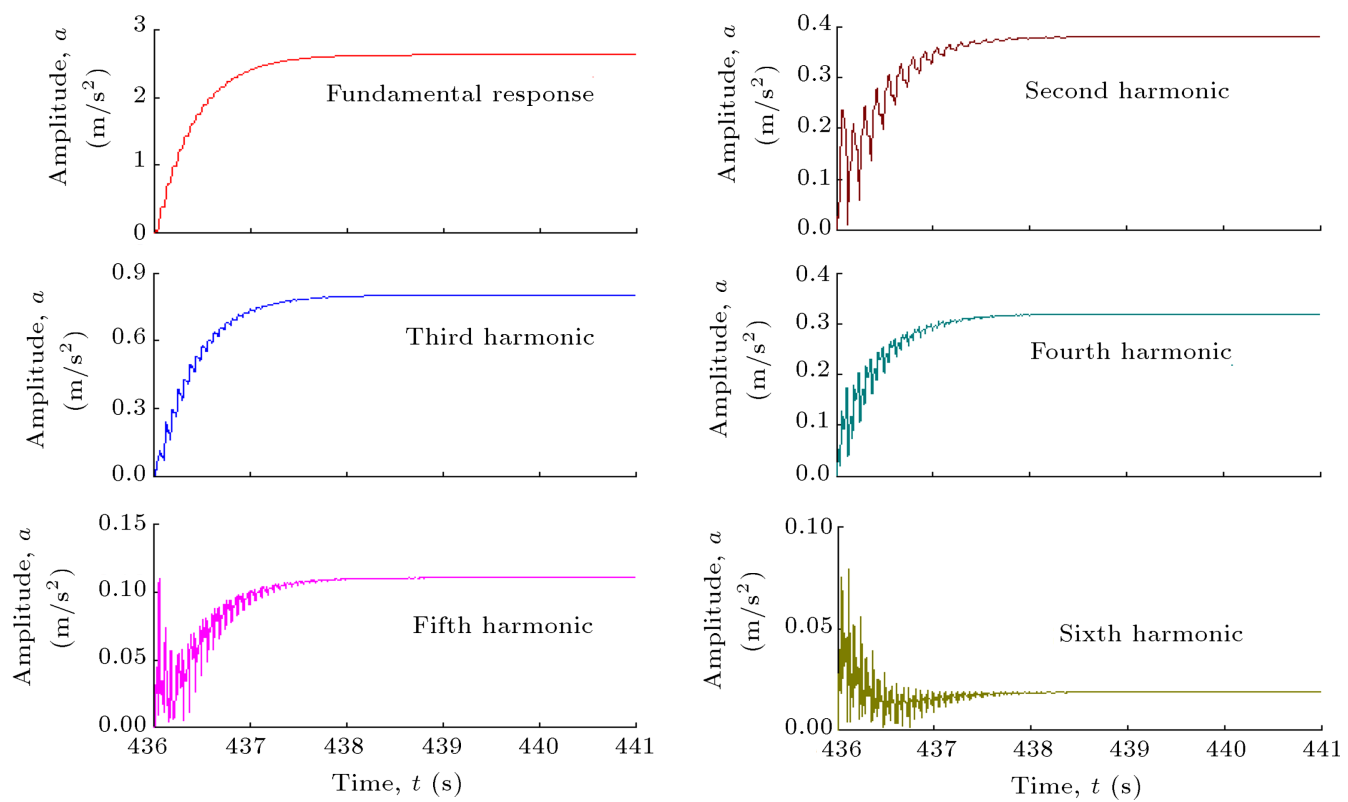

(a) The identified amplitudes
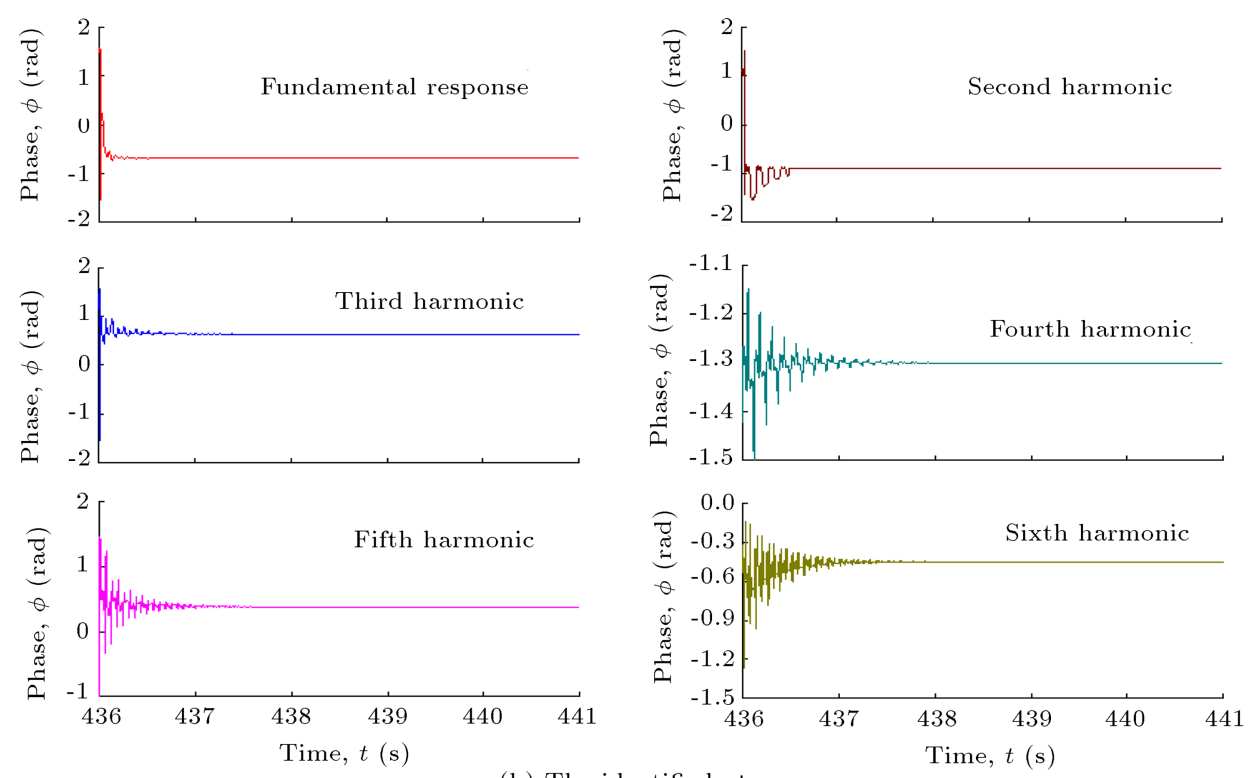

(b) The identified phases

Figure 13. The identified amplitude and phase of each harmonic.

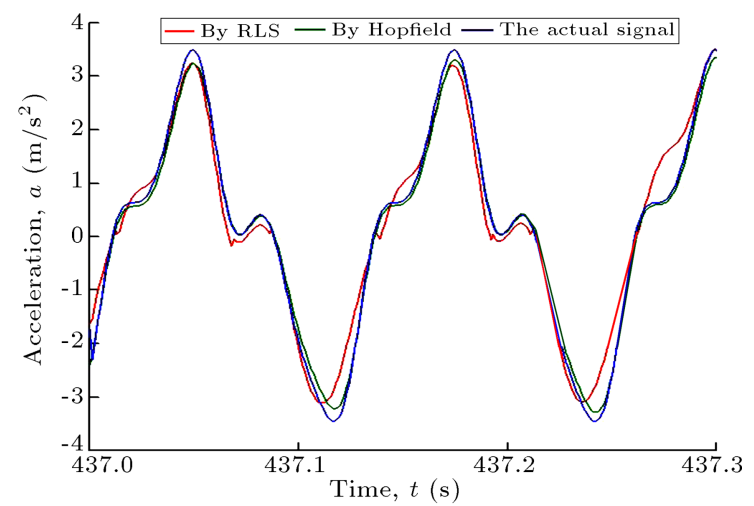

Figure 14. Waveform comparison results with RLS and Hopfield methods.

\section{Conclusions}

To effectively estimate harmonics, a harmonic identification method is developed for a hydraulic shaking table by using Hopfield neural network, whose weights represent harmonics' amplitudes and phases. As the weights of the network are time-varying, the estimator is able to adaptively track the harmonics with amplitudes and phases. The Hopfield network is a finite dimensional system with a recursive structure, which makes a digital control system suitable for the implementation of the developed harmonic identification.

Experiments are performed on the shaking table to verify the proposed harmonic identification. The 

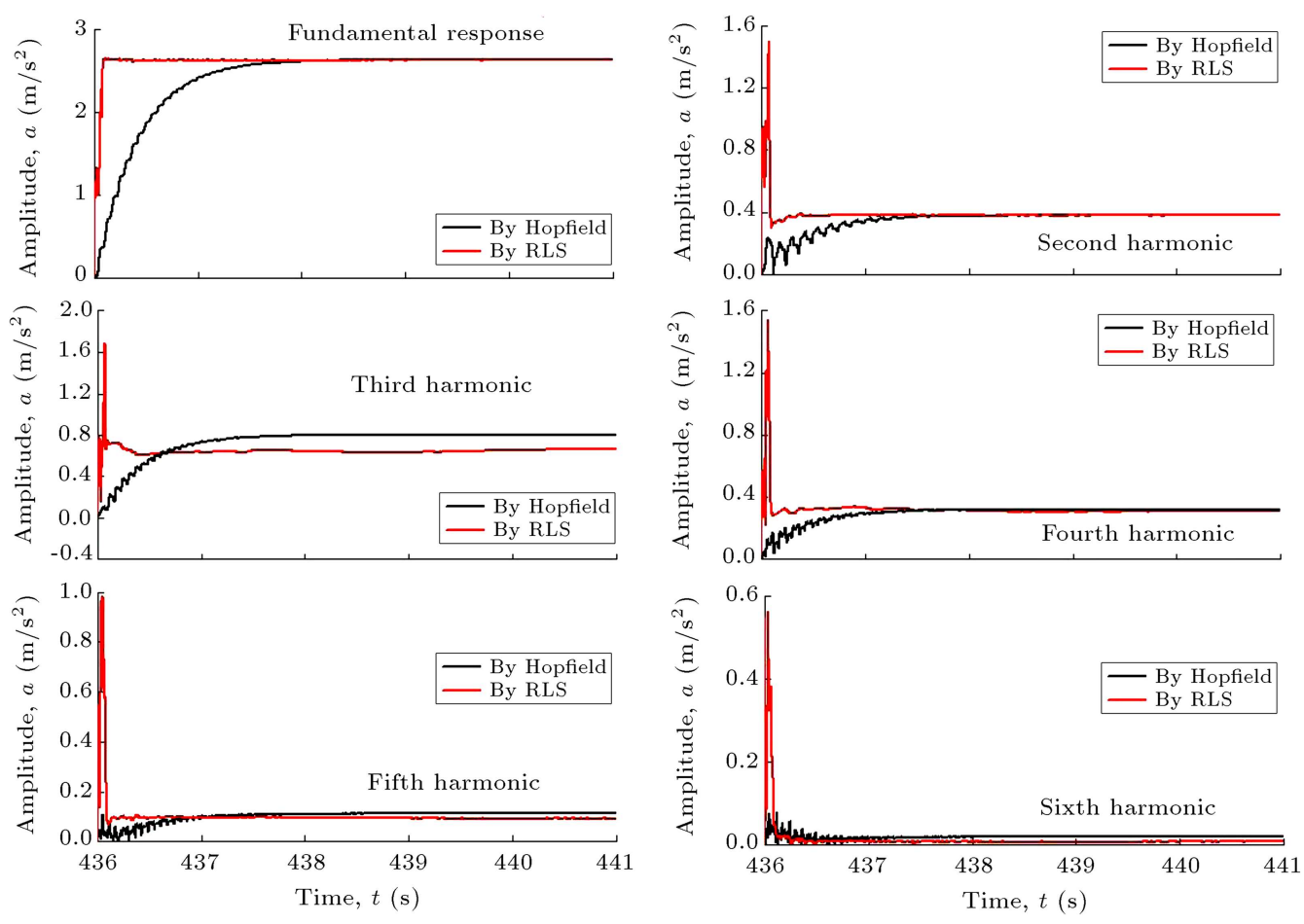

Figure 15. Comparison of identified amplitudes.
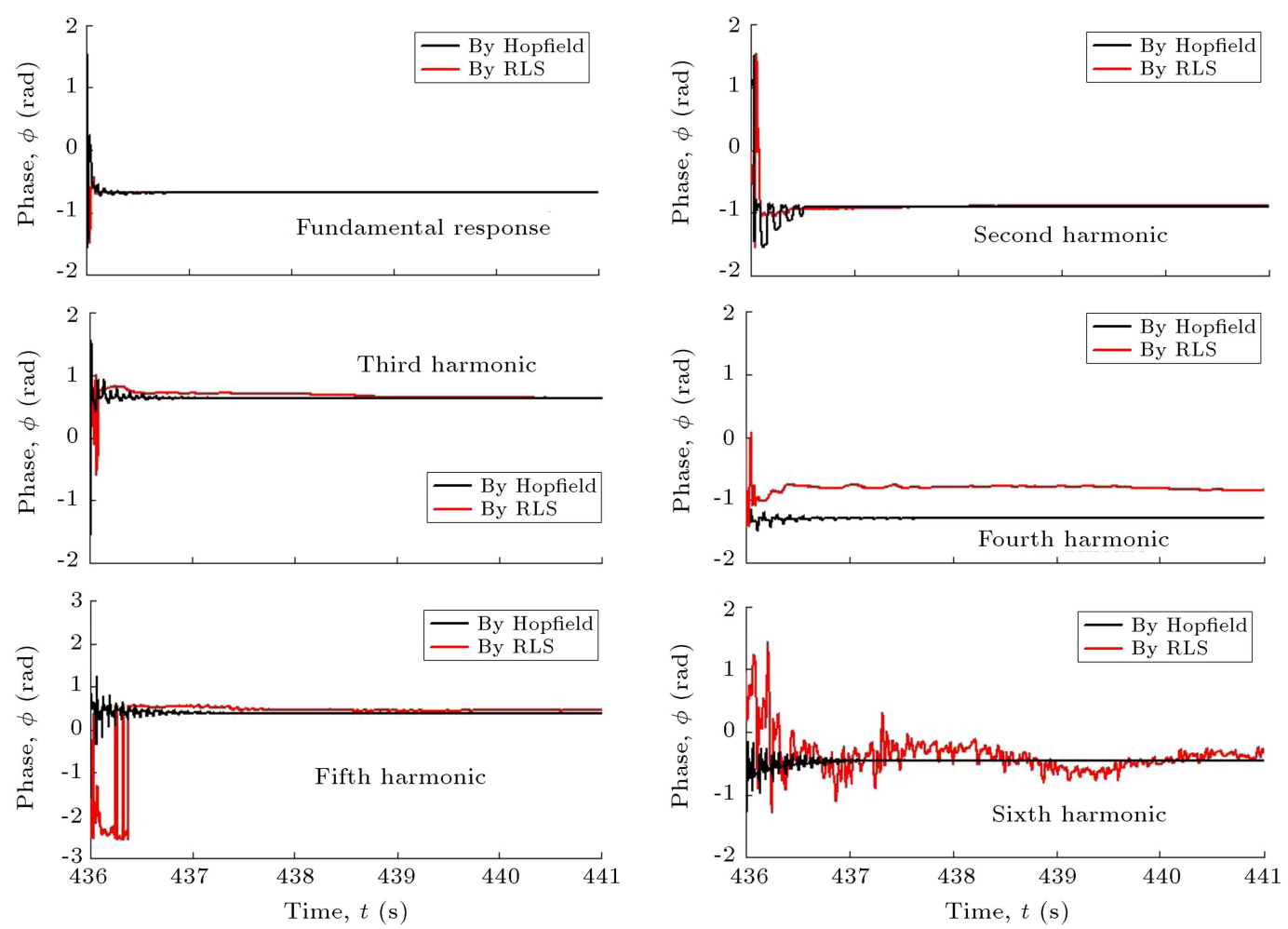

Figure 16. Comparison of identified phases.

estimation results show that identification of amplitude is better than that of phase, and the convergence performance is kept well even for the harmonics which are in the lowest dominance among all harmonics. The estimated results are compared with the THD analysis results, and the estimation error is used to demonstrate the estimation accuracy. The accuracy and real-time performance of the acceleration harmonic identification are then testified.

Compared to DFT or FFT, the proposed identifi- 
cation method operates in time domain and has better real-time performance, so the algorithm can be easily used for real-time harmonic identification. Further comparison studies with RLS-based method are also made to show its good identification performance. The identification algorithm has advantages, such as simple structure, good learning ability, high real-time performance, and high speed recognition. It can decompose each harmonic and detect the amplitude and phase of any order of harmonic, including fundamental response. The online estimated harmonic information can be provided as a basis for further harmonic cancellation.

In particular, the developed identification scheme does not require the system's a priori knowledge. Since higher harmonics are in less dominance among all harmonics, the harmonic estimation is usually up to the 6th harmonic in practical applications. In the experiment, if $n>6$, the identification performance (such as accuracy) cannot be greatly improved with the increase in $n$; however, the increase can lead to high computational burden. It should be noted that the performance of the identification scheme depends critically on the parameter $\kappa$. If a small value for $\kappa$ is chosen, the adaptation will be slow. On the other side, too large $\kappa$ will lead to very oscillatory weights, even divergence.

\section{Acknowledgments}

This project was supported by National Natural Science Foundation of China (Grant No. 51375102), Natural Science Foundation of Heilongjiang Province of China (Grant No. QC2014C050), Supporting Plan Project for Youth Scholar Backbone of General Colleges and Universities of Heilongjiang Province (Grant No. 1253G020), Supporting Plan Project for Youth Scholar Backbone of Harbin Engineering University, and the Fundamental Research Funds for the Central Universities (Grant No. HEUCFQ20150711).

\section{References}

1. Yao, J.J., Hu, S.H., Fu, W. and Han, J.W. "Impact of excitation signal upon the acceleration harmonic distortion of an electro-hydraulic shaking table", Journal of Vibration and Control, 17(7), pp. 1106-1111 (2011).

2. Chung, Y.L., Nagae, T., Hitaka, T. and Nakashima, M. "Seismic resistance capacity of high-rise buildings subjected to long-period ground motions: E-defense shaking table test", Journal of Structural Engineering, 136(6), pp. 637-644 (2010).

3. Merritt, H.E., Hydraulic Control Systems, New York: Wiley (1967).

4. Lin, H.C. "Intelligent neural network based dynamic power system harmonic analysis", International Con- ference on Power System Technology, Singapore, pp. 244-248 (2004).

5. Wang, X.H., He, Y.G. and Long, Y. "Neural network based high accuracy frequency harmonic analysis in power system", 4th International Symposium on Neural Networks, Nanjing, China, pp. 1006-1014 (2007).

6. Hamad, M.S., Gadoue, S.M. and Williams, B.W. "Harmonic compensation of a six-pulse current source controlled converter using neural network-based shunt active power filter", IET Power Electronics, 5(6), pp. 747-754 (2012).

7. Abdeslam, D.O., Wira, P., Fliellert, D. and Mercklé, J. "Power harmonic identification and compensation with an artificial neural network method", IEEE International Symposium on Industrial Electronics, Montreal, QC, Canada, pp. 1732-1737 (2006).

8. Almaita, E. and Asumadu, J.A. "On-line harmonic estimation in power system based on sequential training radial basis function neural network", Proceedings of the IEEE International Conference on Industrial Technology, Auburn, AL, United states, pp. 139-144 (2011).

9. Arabaci, H. "An artificial neural network approach for sensorless speed estimation via rotor slot harmonics", Turkish Journal of Electrical Engineering and Computer Sciences, 22(4), pp. 1076-1084 (2014).

10. Nascimento, C.F., Oliveira Jr., A.A., Goedtel, A. and Dietrich, A.B. "Harmonic distortion monitoring for nonlinear loads using neural-network-method", $A p$ plied Soft Computing Journal, 13(1), pp. 475-482 (2013).

11. Ulinuha, A., Masoum, M.A.S. and Islam, S. "Hybrid genetic-fuzzy algorithm for volt/var/total harmonic distortion control of distribution systems with high penetration of non-linear loads", IET Generation, Transmission and Distribution, 5(4), pp. 425-439 (2011).

12. Zamanan, N., Sykulski, J. and Al-Othman, A.K. "A digital technique for online identification and tracking of power system harmonics based on real coded genetic algorithm", 6th IASTED International Conference on European Power and Energy Systems, Rhodes, Greece, pp. 144-148 (2006).

13. Xue, Z.H., Du, P.J. and Su, H.J. "Harmonic analysis for hyperspectral image classification integrated with PSO optimized SVM", IEEE Journal of Selected Topics in Applied Earth Observations and Remote Sensing, 7(6), pp. 2131-2146 (2014).

14. Yin, Y.N., Lin, W.X. and Li, W.L. "Estimation amplitude and phase of harmonic based on improved PSO", 8th IEEE International Conference on Control and Automation, Xiamen, China, pp. 826-831 (2010).

15. Yang, X.F. and Wang, J.L. "An analysis method for power system transient signal based on improved prony algorithm and PSO algorithm", International Conference on Information Technology and Applications, Chengdu, China, pp. 209-213 (2013). 
16. Vasumathi, B. and Moorthi, S. "Implementation of hybrid ANNPSO algorithm on FPGA for harmonic estimation", Engineering Applications of Artificial Intelligence, 25(3), pp. 476-483 (2012).

17. Ray, P.K. and Subudhi, B. "Ensemble-Kalman-filterbased power system harmonic estimation", IEEE Transactions on Instrumentation and Measurement, 61(12), pp. 3216-3224 (2012).

18. Singh, S.K., Sinha, N., Goswami, A.K. and Sinha, N. "Several variants of Kalman Filter algorithm for power system harmonic estimation", International Journal of Electrical Power and Energy Systems, 78(1), pp. 793800 (2016).

19. Jatoth, R.K. and Reddy, G.A. "A hybrid GA-adaptive particle swarm optimization based tuning of unscented Kalman filter for harmonic estimation", Lecture Notes in Computer Science, Chennai, India, pp. 380-388 (2010).

20. Ren, J. and Kezunovic, M. "A wavelet method for power system frequency and harmonic estimation", North American Power Symposium, Arlington, TX, United states, pp. 1052-1056 (2010).

21. Jain, S.K., Saxena, D. and Singh, S.N. "Adaptive wavelet neural network based harmonic estimation of single-phase systems", Proceedings of the 2011 International Conference and Utility Exhibition on Power and Energy Systems: Issues and Prospects for Asia, Pattaya City, Thailand, pp. 1113-1117 (2012).

22. Yang, J.N. and Cheng, L. "A wavelet model for online tracking of power system: Time-varying harmonics using Kaman filtering", International Conference on Informationization, Automation and Electrification in Agriculture, Zhengjiang, China, pp. 351-358 (2008).

23. Yao, J.J., Jiang, G.L., Di, D.T. and Liu, S. "Acceleration harmonic identification for an electro-hydraulic servo shaking table based on the normalized leastmean-square adaptive algorithm", Journal of Vibration and Control, 19(1), pp. 47-55 (2013).

24. Yao, J.J., Di, D.T., Jiang G.L. and Gao, S. "Acceleration amplitude-phase regulation for electro-hydraulic servo shaking table based on LMS adaptive filtering algorithm", Journal of Vibration and Control, 85(10), pp. 1581-1592 (2012).

25. Yao, J.J., Zhang, L., Chen, S., Wan, Z.S., Wang, T. and Niu, Q.T. "Translational shaking tests achieved on a 6-DOF hydraulic parallel manipulator", Industrial Robot: An International Journal, 43(1), pp. 103-111 (2016).

26. Hillar, C., Mehta, R. and Koepsel, K. "A hopfield recurrent neural network trained on natural images performs state-of-the-art image compression", IEEE International Conference on Image Processing, Paris, France, pp. 4092-4096 (2014).

27. Martin, T.H., Neural Network Design, Beijing: China Machine Press (2007).
28. Singh, S.K., Nath, A., Chakraborty, R., Kalita, J., Sinha, N. and Goswami, A.K. "Fast transverse-RLS algorithm based power system harmonic estimation", 2014 International Conference on Information Communication and Embedded Systems, Chennai, India, pp. 1253-1257 (2015).

29. Garanayak, P., Panda, G. and Ray, P.K. "Harmonic estimation using RLS algorithm and elimination with improved current control technique based SAPF in a distribution network", International Journal of Electrical Power and Energy Systems, 73(12), pp. 209-217 (2015).

30. Ray, P.K. and Subudhi, B. "BFO optimized RLS algorithm for power system harmonics estimation", Applied Soft Computing Journal, 12(8), pp. 1965-1977 (2012).

\section{Biographies}

Jianjin Yao received his PhD degree from the School of Mechanical and Electrical Engineering at Harbin Institute of Technology, China. He is a Professor in the College of Mechanical and Electrical Engineering at Harbin Engineering University. His research interests include electro-hydraulic servo systems, control theory and application, motion simulator, robot technology, hydraulic shaking tables, etc.

Tao Wang is currently finishing his studies for MS degree in Mechanical Engineering at Harbin Engineering University, which he started in 2014. His research interests include the mechanical control and the hydraulic system of electro-hydraulic shaking tables.

Zhenshuai Wan is currently finishing his studies for PhD degree in Mechanical Engineering at Harbin Engineering University. His research interests include electro-hydraulic shaking tables, adaptive control, etc.

Shuo Chen is currently finishing his studies for MS degree in Mechanical Engineering at Harbin Engineering University. His research interests include modal analysis, mechatronics, controller design, etc.

Qingtai Niu is currently finishing his studies for MS degree in Mechanical Engineering at Harbin Engineering University. His research interests include shaking tables, adaptive filtering algorithms, etc.

Le Zhang is currently finishing his studies for MS degree in Mechanical Engineering at Harbin Engineering University. His research interests include shaking tables, mechanical dynamic analysis, etc. 